\title{
Teletrabajo en el contexto Covid-19 y su impacto en la salud de docentes universitarios
}

\author{
Teleworking in the context of Covid-19 and its impact on the health of university teachers \\ Teletrabalho no contexto da Covid-19 e seu impacto na saúde de professores universitários
}

\section{Silvia Elizabet Reyes Narváez ${ }^{1}$}

sreyesn@unasam.edu.pe

https://orcid.org/0000-0002-9624-1997

Bibiana María León Huerta ${ }^{1}$

bibianamarialh@hotmail.com

https://orcid.org/0000-0003-0849-3834

Olga Giovanna Valderrama Rios ${ }^{2}$

ogvalderramar@unac.edu.pe

https://orcid.org/0000-0003-1906-7101

\author{
Llermé Nuñez Zarazú ${ }^{1}$ \\ opebri@yahoo.com \\ https://orcid.org/0000-0002-8011-5072 \\ Ursula Lezameta Blas ${ }^{1}$ \\ urlezab@hotmail.com \\ https://orcid.org/0000-0003-0471-9031
}

\author{
Ponte Valverde Segundo Ignacio ${ }^{3}$ \\ tenpo_01@hotmail.com \\ https://orcid.org/0000-0003-4199-6660
}

${ }^{1}$ Universidad Nacional Santiago Antúnez de Mayolo, Perú
${ }^{2}$ Universidad Nacional del Callao, Perú
${ }^{3}$ Universidad San Ignacio De Loyola, Perú

Recibido 13 de septiembre 2021 / Arbitrado y aceptado 22 de octubre 2021 / Publicado 30 de diciembre 2021

\begin{abstract}
RESUMEN
El teletrabajo es una modalidad de trabajo impulsado en época del contexto Covid-19. Implica la ejecución de labores desde un lugar diferente a la organización haciendo uso de las tecnologías de la información, con ello el estado protege y garantiza el derecho laboral, pero a la vez, constituye una fuente de riesgos para la salud. Objetivo. Evaluar los riesgos laborales del teletrabajo en el marco de la emergencia sanitaria Covid-19 en docentes de una universidad pública y privada. Material y métodos. Investigación básica de diseño no experimental de tipo descriptivo - comparativo. Se realizó en una muestra de 277 docentes de la Universidad Nacional Santiago Antúnez de Mayolo y Universidad San Ignacio de Loyola del Perú. Los datos se recolectaron a través de un Cuestionario de Riesgos Laborales del Teletrabajo aplicado vía online y tuvo 34 ítems. El instrumento evaluó: Riesgos musculoesqueléticos, riesgos psicosociales, fatiga visual y riesgos locativos. Resultados. $48,2 \%$ (54) docentes que realizaron teletrabajo en universidades públicas y $44,2 \%(73)$ de los docentes de universidades privadas presentaron algún riesgo laboral (riesgo musculoesquelético, riesgo psicosocial, riesgo locativo o riesgo visual). El $37,9 \%$ (105) presentaron riesgos laborales músculo esquelético, 22,4\% (62) riesgo locativo, $19,5 \%$ (54) riesgo laboral psicosocial y $8,7 \%(24)$ riesgo visual. Conclusiones. No existe una diferencia significativa de riesgos laborales entre docentes procedentes de universidad pública y privada durante el desarrollo del teletrabajo en el marco de la emergencia sanitaria por Covid-19.
\end{abstract}

Palabras clave: Riesgos laborales; Salud laboral; Condiciones de trabajo; Docentes

\begin{abstract}
Teleworking is a work modality promoted during the Covid-19 context. It implies the execution of work from a place other than the organization making use of information technologies, with this the state protects and guarantees labor law, but at the same time, constitutes a source of health risks. Objective. To evaluate the occupational risks of teleworking in the framework of the Covid-19 health emergency in teachers of a public and private university. Material and methods. Basic research of non-experimental descriptive-comparative design. It was carried out in a sample of 277 teachers from the Santiago Antúnez de Mayolo National University and the San Ignacio de Loyola University of Peru. The data was collected through a Telework Occupational Risks Questionnaire applied online and had 34 items. The instrument evaluated: musculoskeletal risks, psychosocial risks, visual fatigue and locative risks. Results. 48.2\% (54) teachers who teleworked in public universities and $44.2 \%(73)$ of teachers from private universities presented some occupational risk (musculoskeletal risk, psychosocial risk, locative risk or visual risk). $37.9 \%$ (105) presented musculoskeletal occupational risks, $22.4 \%(62)$ locative risk, $19.5 \%(54)$ psychosocial occupational risk and $8.7 \%(24)$ visual risk. Conclusions. There is no significant difference in occupational risks between teachers from public and private universities during the development of telework in the framework of the health emergency caused by Covid-19.
\end{abstract}

Key words: Occupational risks; Occupational health; Working conditions; Professor 


\section{RESUMO}

O teletrabalho é uma modalidade de trabalho promovida no contexto da Covid-19. Implica a execução do trabalho a partir de um local distinto da organização que faz uso das tecnologias de informação, com isso o Estado protege e garante o direito do trabalho, mas ao mesmo tempo constitui uma fonte de riscos para a saúde. Objetivo. Avaliar os riscos ocupacionais do teletrabalho no âmbito da emergência sanitária Covid-19 em docentes de uma universidade pública e privada. Material e métodos. Pesquisa básica de delineamento descritivocomparativo não experimental. Foi realizado em uma amostra de 277 professores da Universidade Nacional Santiago Antúnez de Mayolo e da Universidade San Ignacio de Loyola do Peru. Os dados foram coletados por meio de um Questionário de Riscos Ocupacionais de Teletrabalho aplicado online e continha 34 itens. O instrumento avaliou: riscos musculoesqueléticos, riscos psicossociais, fadiga visual e riscos locativos. Resultados. $48,2 \%$ (54) professores que faziam teletrabalho em universidades públicas e $44,2 \%$ (73) dos professores de universidades privadas apresentaram algum risco ocupacional (risco musculoesquelético, risco psicossocial, risco locativo ou risco visual). $37,9 \%$ (105) apresentavam risco ocupacional musculoesquelético, 22,4\% (62) risco locativo, $19,5 \%$ (54) risco ocupacional psicossocial e $8,7 \%$ (24) risco visual. Conclusões. Não existe diferença significativa nos riscos ocupacionais entre docentes de universidades públicas e privadas durante o desenvolvimento do teletrabalho no quadro da emergência sanitária provocada por Covid- 9.

Palavras-chave: Riscos laborais; saúde Ocupacional; condições de trabalho; professores

\section{INTRODUCCIÓN}

Actualmente la sociedad enfrenta la pandemia del síndrome respiratorio agudo causado por el coronavirus SARS-CoV-2 (Covid-19). En Perú, luego del primer caso confirmado, el gobierno expidió diversos textos normativos como el Decreto de Urgencia $N^{\circ}$ 026-2020 en el cual se establecen algunas medidas excepcionales y temporales para prevenir la propagación del Covid-19, entre ellas las de materia laboral, cambiando las labores presenciales a la modalidad virtual.

La pandemia por Covid-19 ha causado un cambio sin precedentes en el modelo educativo. La educación ha tenido que reinventarse para continuar con el proceso enseñanza-aprendizaje en las instituciones de nivel superior, generando nuevas exigencias al docente universitario como el uso deestrategias didácticas online y el manejo de plataformas virtuales (1). En este contexto de emergencia sanitaria, el Ministerio de Educación permite el desarrollo de la enseñanza superior a través del teletrabajo o virtualización. Este cambio significa adaptar el trabajo presencial a teletrabajo desde el hogar (2).

El teletrabajo es una forma de trabajo que utiliza las tecnologías de información y telecomunicación. Es una opción laboral que protege los derechos de la persona (3). La Ley $N^{\circ} 30036$ define el teletrabajo como la labor que realiza el trabajador, a través de medios informáticos, sin la presencia física en la organización con la cual tiene una relación laboral (4).

El teletrabajo ofrece una alternativa ante los eventos inesperados, pero tiene desventajas para la salud durante su implementación. Según la Organización Iberoamericana de Seguridad Social (OISS), el teletrabajo constituye una fuente de riesgos concretos que es preciso considerar. Trastornos musculoesqueléticos, cuya aparición de estos riesgos son las posturas incorrectas y la prolongada de posturas de sedestación. Fatiga visual como consecuencia una inadecuada luminosidad, la calidad de la pantalla y de la ubicación del equipo informático. Riesgo psicosocial, el hecho de trabajar en casa provoca un cierto aislamiento de los compañeros de trabajo, además el entorno familiar puede afectar psicológicamente al teletrabajador por las tensiones que produce el exceso de horas de trabajo (5). De igual 
modo, el riesgo locativo se produce cuando en casa no hay un espacio exclusivo para trabajar, la línea que separa el trabajo de la vida personal es imperceptible. Lo adecuado es que el espacio tenga luz natural, poco ruido, ventilación y temperatura adecuada (6).

Este modo de trabajo es aún poco estudiado y considerado en la legislación de algunos países. Sin embargo, en la actualidad su uso cada vez más frecuente, no se ve reflejado en la prevención de riesgos laborales (5). El teletrabajo puede implicar riesgos para la salud del trabajador como la fatiga visual, estrés, deformación musculoesquelética, fatiga muscular, aislamiento, invasión a la privacidad, entre otros (7).

El sistema educativo en las universidades de México es uno de los más dotados en equipamiento para el servicio a distancia y ha permitido a los docentes desplegar sus habilidades para continuar con su trabajo (2). En la Universidad de Juárez, los docentes realizan teletrabajo por horas, la edad promedio es $45,7 \%$ años, $58 \%$ son mujeres, $62,8 \%$ han tenido experiencias previas en actividades sincrónicas; asimismo, todos cuentan con equipos tecnológicos en casa como computadoras o tabletas. Sobre el espacio disponible $25,6 \%$ utiliza el comedor, lo que indica que, a pesar de contar con todos los recursos tecnológicos, los docentes no cuentan con un espacio para realizar el teletrabajo en tiempos de Covid 19 (2). Corrales, demostró que las actitudes hacia el teletrabajo en docentes de una universidad pública, están determinadas por el contacto interpersonal, manejo del tiempo, recursos, espacio y uso de la tecnología (8).
El riesgo psicosocial por la enseñanza a través de teletrabajo en Ecuador se encontró síntomas de cansancio, irritabilidad, ansiedad, insomnio, mareos, falta de atención (9). En otros resultados se observó un estrés técnico que se manifiesta por desajustes de la persona con la organización, la tecnología y la propia persona a causa del desequilibrio con el entorno educativo (10). La relación del uso de la TIC y las enfermedades musculoesqueléticas y malestar visual en trabajadores españoles, se reportó que la mayor incidencia de trastornos visuales y musculoesqueléticos se debe al uso prolongado de las computadoras portátiles y de escritorio (11). En universidades peruanas se encontró $100 \%$ de prevalencia de trastornos musculoesqueléticos en docentes que realizan teletrabajo. De ellos, $67,27 \%$ tiene trastornos a nivel dorso lumbar y 64,55\% a nivel del cuello. Las jornadas laborales oscilan más de 10 horas (39,09\%) y trabajan de 5 a 7 días (82,73\%) (12).

En el contexto de la emergencia sanitaria es importante conocer los riesgos laborales del teletrabajo en docentes universitarios, ya que permitiría desarrollar estrategias para la prevención y atención oportuna de los riesgos musculoesqueléticos, psicosociales, locativos y visuales. Asimismo, a partir de esta experiencia orientar a las instituciones en la elaboración de protocolos para la prevención de riesgos laborales ocasionados por el teletrabajo.

El objetivo del estudio es evaluar los riesgos laborales en el teletrabajo en el marco de la emergencia sanitaria Covid-19 en docentes de una universidad pública y privada de Perú. 


\section{MATERIALES Y MÉTODOS}

Estudio descriptivo-comparativo de diseño no experimental realizado durante los meses de abril a diciembre del 2020 en dos universidades peruanas. La población estuvo compuesta por docentes de la Universidad Nacional Santiago Antúnez de Mayolo (UNASAM) de la región Ancash y docentes de la Universidad San Ignacio de Loyola (USIL) de la Región Lima. La muestra estuvo conformada 165 docentes de la Universidad pública y 112 docentes de la Universidad privada, la selección de las unidades muestrales fue por un tipo de muestreo por "cuotas".

Los criterios de inclusión consideraron a docentes que trabajan en la UNASAM y USIL, de ambos sexos, edad de 30 a 70 años y que acepten participar voluntariamente. Los Criterios de exclusión fueron a docentes que no hacen uso del teletrabajo, docentes que no acepten participar y docentes mayores de 70 años.

Para la recolección de datos se utilizó la técnica de encuesta y como instrumento un Cuestionario de Riesgos Laborales en el Teletrabajo, el cual tuvo dos partes: a) Datos generales que incluye características sociodemográficas de los docentes universitarios como edad, sexo, condición laboral, centro laboral, años de servicio. b) Riesgos laborales: con ítems que utiliza la Organización Iberoamericana de Seguridad Social adaptado por los investigadores y tuvo 34 ítems. Este último instrumento evaluó cuatro tipos de riesgos laborales del teletrabajo: Riesgos musculoesqueléticos, riesgos psicosociales, fatiga visual y riesgos locativos (5).

El cuestionario fue aplicado vía online, validado a través de juicio de expertos y confiable mediante la prueba de Alfa de Cronbach $(\alpha=892)$. Según las respuestas en cada dimensión, se consideró que existe riesgo laboral cuando las respuestas sean negativas en un $50 \%$. Los datos se procesaron en el paquete SPSS-22 y para la prueba de hipótesis se utilizó diferencia de proporciones, también se usó la estadística descriptiva presentando los resultados en tablas y gráficos.

El presente estudio consideró los principios éticos establecidos por la Declaración de Helsinki como la beneficencia, no maleficencia, justicia y autonomía. Asimismo, antes de la aplicación del test se solicitó el consentimiento informado a cada docente aceptando su participación. De igual modo el estudio fue aprobado por el Comité de ética de la UNASAM.

\section{RESULTADOS Y DISCUSIÓN}

En la Tabla 1, donde se presentan las características sociolaborales de los docentes universitarios del estudio, se aprecia que la mayoría (34,3\%) tienen una edad comprendida entre 41 y 50 años, $63,2 \%$ son de sexo masculino, 59,6\% labora en el sector público, $48,1 \%$ trabaja entre 5 a 10 horas diarias y el $67,5 \%$ tiene menos de 1 año de servicio. 
Tabla 1. Características sociolaborales de docentes universitarios en teletrabajo en el contexto COVID - 19.

\begin{tabular}{|c|c|c|}
\hline Características & Frecuencia & $\%$ \\
\hline \multicolumn{3}{|l|}{ Edad } \\
\hline $30-40$ & 76 & 27,4 \\
\hline $41-50$ & 95 & 34,3 \\
\hline $51-60$ & 62 & 22,4 \\
\hline$>61$ años & 44 & 15,9 \\
\hline \multicolumn{3}{|l|}{ Sexo } \\
\hline Femenino & 102 & 36,8 \\
\hline Masculino & 175 & 63,2 \\
\hline \multicolumn{3}{|l|}{ Condición laboral } \\
\hline Nombrado & 114 & 41,2 \\
\hline Contratado & 163 & 58,8 \\
\hline \multicolumn{3}{|l|}{ Centro laboral } \\
\hline Público & 165 & 59,6 \\
\hline Privado & 112 & 40,4 \\
\hline \multicolumn{3}{|l|}{ Horas de trabajo a la semana } \\
\hline Menos de 5 hrs & 6 & 2,2 \\
\hline de 5 a $10 \mathrm{hrs}$ & 122 & 44 \\
\hline Más de 11 hrs & 149 & 53,8 \\
\hline \multicolumn{3}{|l|}{ Tiempo de servicio } \\
\hline Menos de 1 año & 22 & 7,9 \\
\hline de 1 a 10 años & 153 & 55,2 \\
\hline de 11 a 22 años & 53 & 19,1 \\
\hline de 23 años a más & 49 & 17,7 \\
\hline Total & 277 & 100 \\
\hline
\end{tabular}

En la Tabla 2, se muestra el riesgo laboral de los docentes universitarios según el tipo de universidad donde laboran en teletrabajo en el contexto COVID-19. Se observa que del total de docentes que laboran en una universidad privada el $51,8 \%$ no presentó riesgo laboral en el teletrabajo y el $48,2 \%$ si presentó riesgo laboral. Con respecto a los docentes que laboran en una universidad pública se aprecia que un $55,8 \%$ no presento riesgo laboral en el teletrabajo y un $44,2 \%$ de docentes si presento riesgo laboral. Realizando el análisis estadístico, no existe una diferencia significativa de riesgos laborales entre docentes de universidad pública y privada durante el desarrollo del teletrabajo en el marco de la emergencia sanitaria Covid-19 $(P=0.515)$. 
Tabla 2. Riesgo laboral de docentes universitarios según tipo de universidad, en teletrabajo en el contexto COVID-19.

\begin{tabular}{lccccccc}
\hline \multirow{2}{*}{ Riesgo Laboral } & \multicolumn{2}{c}{ Universidad Privada } & \multicolumn{2}{c}{ Universidad Pública } & Dif. & Z & P \\
& $\mathbf{N}^{\circ}$ & $\mathbf{\%}$ & $\mathbf{N}^{\circ}$ & $\mathbf{\%}$ & & \multirow{2}{*}{0,515} \\
\hline Presentan & 54 & $48,2 \%$ & 73 & $44,2 \%$ & $4 \%$ & 0,65 & \\
No presentan & 58 & $51,8 \%$ & 92 & $55,8 \%$ & & & \\
\hline Total & $\mathbf{1 1 2}$ & $\mathbf{1 0 0}$ & $\mathbf{1 6 5}$ & $\mathbf{1 0 0}$ & & & \\
\hline
\end{tabular}

De los riesgos musculo esqueléticos presentados en la Tabla 3, hallados en los docentes universitarios que realizan teletrabajo en el contexto COVID-19, se infiere que el $81,6 \%$ de docentes dispone de espacio suficiente para apoyar las manos y/o antebrazo delante del teclado, en cuanto al espacio dispuesto para el teletrabajo, $75,5 \%$ de los encuestados respondieron que disponen de un espacio suficiente para adoptar una postura cómoda, con respecto a la silla que utiliza para el teletrabajo, $81,2 \%$ de docentes manifestó que no utiliza una silla con cinco apoyos, $74,4 \%$ manifestó que el espaldar de la silla no es regulable en inclinación, 70,4\% manifestó que el respaldo de la silla no es regulable en altura independientemente del asiento y el $53,1 \%$ no cuenta con una silla regulable en altura.

Tabla 3. Riesgo musculoesqueléticos de docentes universitarios en teletrabajo en el contexto COVID-19

\begin{tabular}{|c|c|c|c|c|c|c|c|}
\hline & \multirow{2}{*}{ RIESGO MUSCULO ESQUELÉTICO } & \multicolumn{2}{|c|}{ SI } & \multicolumn{2}{|c|}{ NO } & \multicolumn{2}{|c|}{ TOTAL } \\
\hline & & $\mathbf{N}^{\circ}$ & $\%$ & $\mathbf{N}$ & $\%$ & $\mathbf{N}^{\circ}$ & $\%$ \\
\hline 1 & $\begin{array}{l}\text { Tiene espacio suficiente para apoyar las manos y/o } \\
\text { antebrazos delante del teclado }\end{array}$ & 226 & 81,6 & 51 & 18,4 & 277 & 100 \\
\hline 2 & $\begin{array}{l}\text { Tiene espacio suficiente, debajo de la superficie de trabajo, } \\
\text { para favorecer una postura cómoda }\end{array}$ & 209 & 75,5 & 68 & 24.5 & 277 & 100 \\
\hline 3 & Utiliza una silla con cinco apoyos & 52 & 18,8 & 225 & 81,2 & 277 & 100 \\
\hline 4 & La silla es regulable en altura & 130 & 46,9 & 147 & 53,1 & 277 & 100 \\
\hline 5 & El espaldar de la silla es regulable en inclinación & 71 & 25,6 & 206 & 74,4 & 277 & 100 \\
\hline 6 & $\begin{array}{l}\text { El espaldar de la silla es regulable en altura } \\
\text { independientemente del asiento }\end{array}$ & 82 & 29,6 & 195 & 70,4 & 277 & 100 \\
\hline
\end{tabular}


Los riesgos psicosociales presentados por los docentes universitarios que realizan teletrabajo en el contexto COVID-19 mostrados en el Gráfico 1, se aprecia que el $72,9 \%$ de profesores no están informados sobre los riesgos del teletrabajo, $54,2 \%$ no conoce los procedimientos y normas en lo referente a trabajo seguro y saludable, $43,0 \%$ tampoco conocen el procedimiento para sus reportes y entregas de comunicación, 38,3\% no está informado de primeros auxilios frente en caso de emergencias en su trabajo y solo un $36,5 \%$ no conoce las medidas de emergencia en caso sea necesario.

Con respecto a los riesgos locativos presentados en el Gráfico 2, hallados en los docentesuniversitariosque realizanteletrabajo en el contexto COVID-19: $60,3 \%$ de docentes manifestaron que en el cuadro eléctrico de su casa los diferenciales no tienen identificados los circuitos que protegen, $52,7 \%$ indicó que el cuadro eléctrico de su casa no está dentro de un armario de plástico y el $40,1 \%$ indicó que el área no está libre de interrupciones, así mismo $33.2 \%$ manifestó que la instalación eléctrica no tiene un cuadro eléctrico con magneto térmicos y diferenciales que protejan los enchufes, y el $32.5 \%$ manifestó que el área de área de trabajo no tiene privacidad.

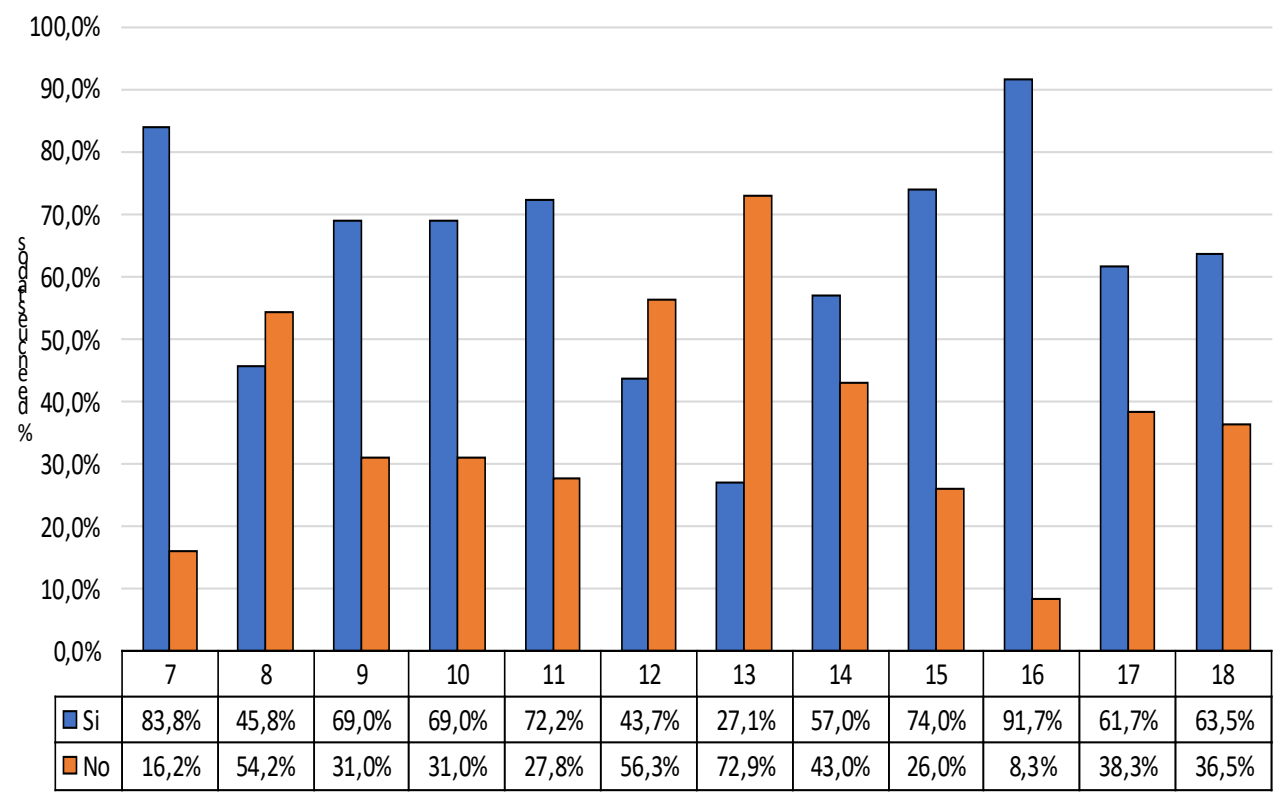

7. El software es fácil y apropiado a la tarea

8. Conoce los procedimientos y normas del teletrabajo, en lo referente a trabajo seguro y saludable

9. Está establecido su disponibilidad de día/hora para establecer contacto con la institución

10. El tiempo de trabajo está señalado

11. Realiza pausas con periodicidad y duración adecuada

12. Tiene dificultad para limitar el tiempo de trabajo por exceso de horarios

13. Está informado de los riesgos del teletrabajo

14. Conoce el procedimiento de comunicación para sus reportes y entregas a realizar

15. Sabe cómo solicitar consultas y orientación en el uso de TICS y equipos

16. Sabe cómo comunicarse con sus representantes de la institución

17. Está informado en primeros auxilios en caso de emergencias

18. Conoce las medidas de emergencia en caso sea necesario.

Gráfico 1. Riesgos psicosociales de docentes universitarios en teletrabajo en el contexto COVID-19. 


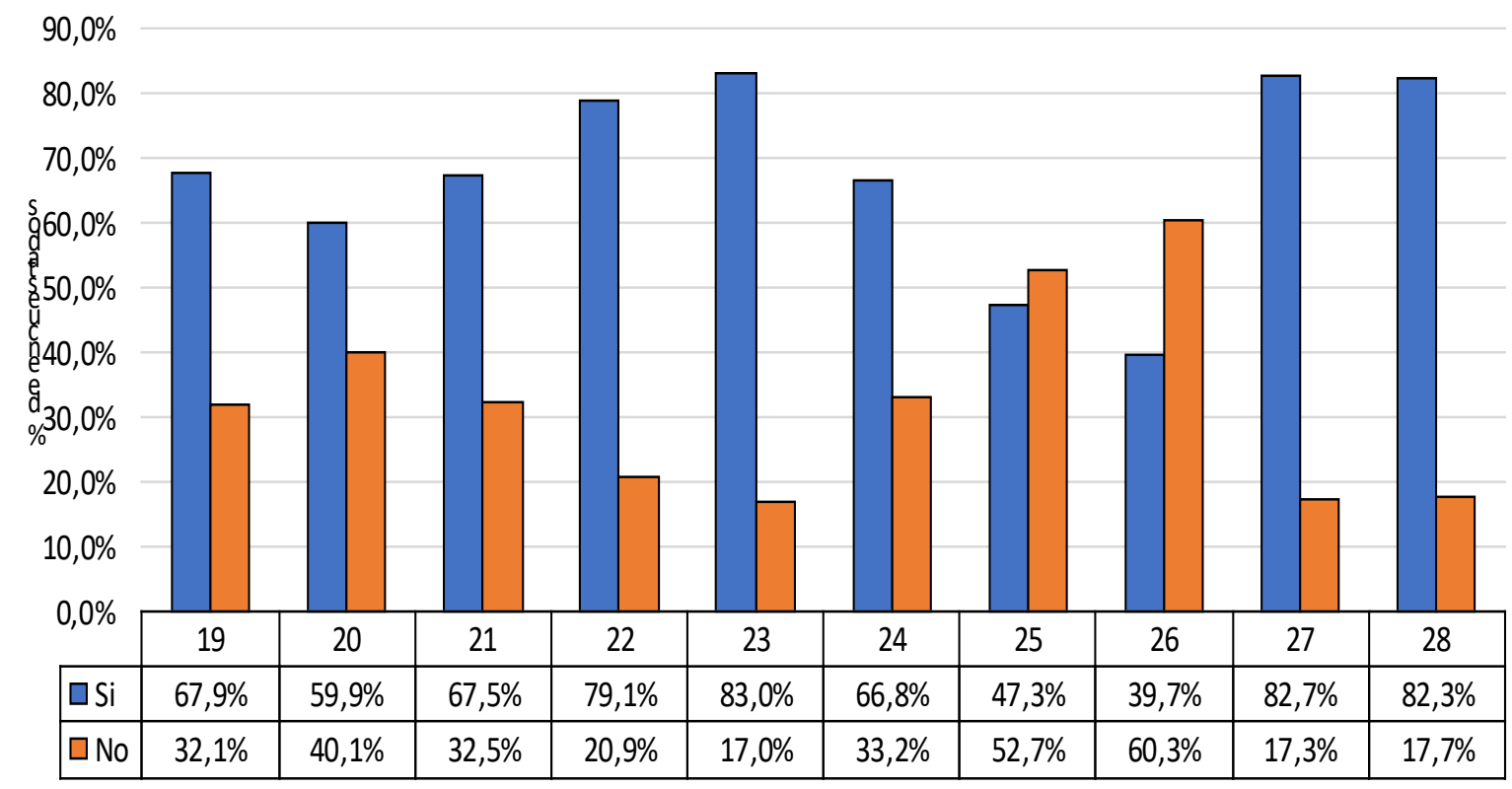

7. Tiene espacio suficiente para realizar su trabajo en forma seguras $\left(2 \mathrm{~m}^{2}\right.$, de superficie libre o $10 \mathrm{~m}^{3}$ no ocupados $)$.

8. El espacio de trabajo está libre de interrupciones

9. El área de trabajo tiene privacidad

10. El cableado de su ordenador cumple con no atravesar las zonas de acceso de su trabajo

11. Los enchufes y cables están en buenas condiciones

12. La instalación eléctrica tiene un cuadro eléctrico con magnetotérmicos y diferenciales que protegen los enchufes

13. El cuadro eléctrico está dentro de un armario de plástico (Si fuera de metal está conectada a tierra)

14. Los diferenciales del cuadro eléctrico tienen identificados los circuitos que protegen

15. La temperatura del ambiente tiene ventilación adecuada

16. La superficie de trabajo es suficiente para ubicar todos los elementos de trabajo (pantalla, teclado, agendas, etc.)

Gráfico 2. Riesgos locativos de docentes universitarios en teletrabajo en el contexto.

\section{COVID-19}

Finalmente, en el Gráfico 3, muestran los riesgos de fatiga visual presentados por los docentes universitarios que realizan teletrabajo en el contexto COVID-19, donde el 33,2\% manifestó que la pantalla no está alejada de la ventana o foco de luz, $32,5 \%$ de docentes afirmó que la superficie de trabajo no es de color mate y $40,1 \%$ afirmó que el brillo de la pantalla no es ajustable. 


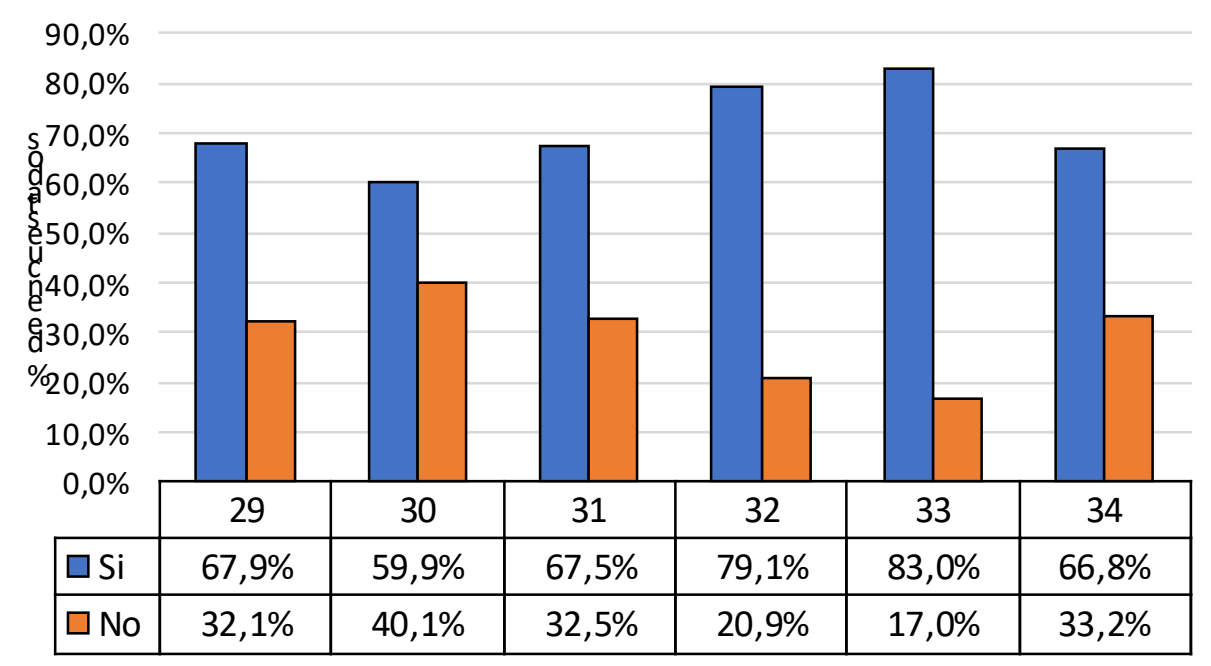

7. La imagen de la PC es estable, sin fluctuaciones ni parpadeos

8. El brillo y contraste de la PC son ajustables

9. La superficie de trabajo es mate

10. La pantalla de la PC es independiente del teclado y tiene regulación en inclinación y en giro.

11. La superficie de trabajo tiene iluminación adecuada para leer sin dificultad los archivos.

12. La pantalla no está situada de frente o de espaldas a una ventana o foco de luz exterior.

Gráfico 3. Riesgos visuales de docentes universitarios en teletrabajo en el contexto COVID-19.

\section{Discusión}

Los docentes universitarios vienen desarrollando el teletrabajo como consecuencia de las medidas emanadas por el Ministerio de Educación a consecuencia del estado de emergencia por el Covid-19, con el propósito de evitar la concurrencia de docentes y estudiantes a las instalaciones de las universidades y así evitar el contagio masivo del SARS- CoV-2. Durante el semestre 2020-I los docentes asumieron el desarrollo de las actividades académicas desde sus hogares haciendo uso de sus equipos tecnológicos computador, laptop, Tablet, así como de los servicios de internet, adecuando su espacio personal para el desarrollo de las clases a distancia o teletrabajo.
El teletrabajo, modalidad adoptada por el docente ha generado algunos riesgos en el estado de salud como las obtenidas en la presente investigación. En relación a la caracterización de la población encuestada la mayoría tienen una edad comprendida entre 41 y 50 años, el sexo masculino predomina en comparación a las mujeres en $63,2 \%$. Similares resultados a los obtenidos por García-Salirrosas y Sánchez-Poma, (12) quienes reportan que el grupo etario mayoritariamente fue 39,09\% de 41 a 50 años y el 70,9\% fueron varones y $29,09 \%$ mujeres.

Referentealapresenciaderiesgoslaborales entre universidad pública y universidad privada no hay una diferencia significativa $(P=.515)$, 
esto probablemente a que las condiciones de trabajo son similares en ambas instituciones. La jornada laboral, las actividades académicas, la disponibilidad de tiempos y espacios, así como el uso y disponibilidad de los equipos tecnológicos. Sin embargo, predominan los riesgos musculoesqueléticos en los indicadores evaluados. Estos resultados concuerdan a los reportados por García-Salirrosas y SánchezPoma, (12) que el $100 \%$ de la población encuestada evidencia la prevalencia de los trastornos musculoesqueléticos. Asimismo, Soria y otros, (11) indican que el patrón de uso con la mayor incidencia de trastornos musculoesqueléticos, especialmente en la muñeca y el cuello, combina el uso de computadoras portátiles y de escritorio durante largas horas de trabajo. De igual modo Tejada y Reyes (13), evidenció que los trastornos musculoesqueléticos más comunes son el dolor de columna, en hombros y dedos, todo ello asociado al inadecuado mobiliario y dispositivos, así como la mala postura. En los resultados del estudio el $81,2 \%$ no utiliza una silla con cinco apoyos, $74,4 \%$ no cuenta con una silla con el respaldo regulable en inclinación y $70,4 \%$ no cuenta con sillas que tengan el respaldo regulable en altura independientemente del asiento, siendo factores de riesgo muy importantes que van contribuir en generar molestias musculoesqueléticas en el personal docente universitario.
En cuanto a los riesgos laborales psicosociales la mayoría de docentes no está informado sobre los riesgos del teletrabajo, no conoce los procedimientos y normas del trabajo seguro y saludable, los procedimientos de comunicación para sus reportes y entregas y no está informado de primeros auxilios en caso de emergencias; estos son factores que conducen a los problemas de salud en el aspecto psicosocial; así mismo cabe señalar sumado a otros factores extralaborales puede convertirse en un factor generador de estrés. Tal como señala, Castellanos y otros, (14) el $85 \%$ de los participantes manifestaron una percepción de estrés medio, $23,7 \%$ en alto y el $20 \%$ en muy alto durante el teletrabajo. También Huarcaya, (15) en una revisión descriptiva de los efectos de la pandemia COVID-19 en la salud mental evidenció ansiedad, depresión y estrés en la población en estudio. Para Tejada y Reyes, (13) el trastorno psicosocial se produce por una sobrecarga laboral, el aislamiento social, uso prolongado de trabajo y el incumplimiento de las tareas domésticas, situación que puede ocasionar estrés al teletrabajo.

Con respecto al riesgo laborales locativo, se aprecia que el ambiente donde el docente universitario realiza el teletrabajo no tiene privacidad y no está libre de interrupciones, asimismo; no tiene las condiciones adecuadas en la instalación eléctrica y cuadro eléctrico, dichos factores son conducente al riesgo locativo como consecuencia del teletrabajo 
por confinamiento, como señalan Castellanos y otros, (14) el 3,7\% considera que el ambiente de trabajo en su casa es inadecuado. Satizábal, Polo y Valbuena, (16) sostienen que el uso de las TIC, la organización del trabajo y las condiciones de seguridad como instalaciones eléctricas, son factores que producen alteraciones musculoesqueléticas, Tecnoestrés y accidentes de trabajo. Cabe precisar que los docentes universitarios a falta de un ambiente exclusivo para teletrabajar y con el propósito de cumplir con sus actividades laborales han adecuado su espacio para trabajar en casa generando incomodidad en el resto de los integrantes de la familia.

Finalmente, referente al riesgo de la fatiga visual más del $50 \%$ de docentes manifiesta que la Pc no tiene la ubicación ni las condiciones para el trabajo remoto como: la pantalla no está alejada de la ventana o foco de luz, la superficie de trabajo no es de color mate, la pantalla no es independiente del teclado y no tiene regulación en inclinación y giro, además trabajan entre 5 a 10 horas diarias. Todas estas condiciones predisponen a la fatiga visual por la sobre exposición a las fuentes de luz que posteriormente podrían a manifestarse síntomas como irritación ocular, ojo seco, etc. Como lo reporta Swetha y otros, (17) el 78,33\% (94/120) de los encuestados en los últimos 6 meses, $32,5 \%$ tenía lagrimeo y dolor en los ojos, $27,5 \%$ tenía irritación de ojos y el $25,8 \%$ tenía ardor y picazón en los ojos mientras trabajaba en la computadora. De igual manera, en un estudio realizado en una universidad de Ecuador reportó que el $40 \%$ de docentes que realiza teletrabajo padece síntomas de fatiga visual (ojos rojos, cansancio y pesadez) relacionado a la excesiva exposición a la pantalla de la computadora (18).

Las limitaciones que presentó el estudio fue principalmente para la recolección de datos, debido a la emergencia sanitaria Covid-19 se realizó vía online, pero algunos docentes al inicio no querían realizar el cuestionario por temor a brindar información negativa de la institución.

\section{CONCLUSIONES}

Aproximadamente el $50 \%$ de docentes de universidades públicas y privadas que realizan teletrabajo presentan algún riesgo laboral (riesgo musculoesquelético, riesgo psicosocial, riesgo locativo o riesgo visual). Además, no existe diferencia significativa de riesgos laborales entre docentes de universidad pública y privada durante el desarrollo del teletrabajo en el contexto Covid-19.

Por ello, se recomienda que, para evitar riesgos laborales a consecuencia del teletrabajo, se debe garantizar las condiciones de un mobiliario y dispositivos adecuados, así como las condiciones físicas y el cumplimiento de las normativas de protección al trabajador. 


\section{REFERENCIAS BIBLIOGRÁFICAS}

1. Martínez-Garcés J, Garcés-Fuenmayor J. Competencias digitales docentes y el reto de la educación virtual derivado de la covid-19. Educación y Humanismo [Internet]. 2020 [Citado 11/04/2021]; 22(39):1-16. Disponible en: https://doi.org/10.17081/eduhum.22.39.4114

2. De Los Heros $M$, Murillo $S$, Solana N. Satisfacción laboral en tiempos de pandemia: el caso de docentes universitarios del área de salud. Revista de Economía del Caribe [Internet]. 2020 [Citado 11/11/2020]; 26:121. Disponible en: http://portal.amelica.org/ ameli/jatsRepo/318/3181675005/index.html

3. Valencia A. Aspectos regulatorios del teletrabajo en Perú: análisis y perspectivas. Nueva época [Internet]. 2018 [Citado 16/03/2021]; 12(41): 203-226. Disponible en: https://revistaius.com/index.php/ius/article/ view/319/599

4. Decreto Supremo que aprueba el Reglamento de la Ley que regula el teletrabajo NN30036/2015. Diario el Peruano 2015 [Citado 14/12/2020] Disponible en: https://busquedas. elperuano.pe/normaslegales/decretosupremo-que-aprueba-el-reglamento-de-laley-n-30036-decreto-supremo-n-009-2015tr-1307067-3/

5. Organización Iberoamericana de Seguridad Social. Prevención de riesgos laborales en el teletrabajo. [Internet]. España; 2019. [Citado 14/12/2020]. Disponible en: https://oiss.org/ wp-content/uploads/2019/06/EOSyS-18-PRLen-el-teletrabajo.-doc.pdf

6. Borondo S. 2020. Cuáles son los riesgos del teletrabajo y como prevenirlos. Disponible en: https://www.elcorreo.com/tecnologia/ empresas/riesgos-teletrabajo-prevenirlos20200415113441-nt.html

7. Osio H. Salud y seguridad en el teletrabajo: caso Argentina. Visión gerencial [Internet]. 2015 [Citado 19/01/2021]; 2:410-426. Disponible en: https://www.researchgate.net/ publication/298793001_Salud_y_seguridad_ en_el_teletrabajo_Caso_Argentina
8. Corrales S. Actitud hace el teletrabajo en un grupo de docentes de una Universidad Pública. [Tesis de maestría]. Colombia: Universidad EAFIT; 2019 [Citado 30/01/2021]. Disponible en: https://repository.eafit.edu.co/ handle/10784/15988

9. Terán D, Córdova M, Muquinche J, Gordón P. Evaluación de la carga y fatiga mental en docentes por teletrabajo a causa del COVID-19. Ciencia Digital [Internet]. 2021 [Citado 10/02/2021]; 5(1): 6-14. Disponible en: https:// doi.org/10.33262/cienciadigital.v5i1.1515

10. Wang X, Bo L. Technostress Among University Teachers in Higher Education: A Study Using Multidimensional Person-Environment Misfit Theory. Frontiers in Psychology [Internet]. 2019 [Citado 20/02/2021]; 10 (1791): 1-13. Disponible en: https://doi.org/10.3389/fpsyg.2019.01791

11. Soria M, López J, Torrano f, García G, Lara A. Nuevos patrones de uso de las tecnologías de la información y la comunicación en el trabajo y sus relaciones con el malestar visual y las enfermedades musculoesqueléticas: resultados de un estudio transversal de organizaciones españolas. Res. Salud Pública [Internet]. 2019 [Citado 8/01/2021]; 16; 3166. Disponible en: https://doi.org/10.3390/ ijerph16173166

12. García-Salirrosas E, Sánchez-Poma R. Prevalencia de trastornos musculoesqueléticos en docentes universitarios que realizan teletrabajo en tiempos de COVID-19. Anales De La Facultad De Medicina [Internet]. 2020 [Citado 8/03/2021]; 81(3): 301-307. Disponible en:

https://doi.org/10.15381/anales. v81i3.18841

13. Tejada B, Reyes L. Teletrabajo, impactos en la salud del talento humano en época de pandemia. Revista Colombiana de Salud Ocupacional. [Internet]. 2021; 11 (2): e-6553. http://dx.doi.org/10.18041/2322-634X/ rcso.2.2021.6553

14. Castellanos A, Quintan P, Carvajal J, Vargas I. Relación de estrés y el trabajo mediante sistemas durante el periodo de confinamiento 
en docentes y personal administrativo de una Institución de Educación Superior. Revista Ibérica de Sistemas e Tecnologías de Información RISTI [Internet]. 2021 [Citado 28/02/2021]; E39: 325-340. Disponible en https://search.proquest.com/openview/ $32 \mathrm{f} 793$ ec5f4a1489ed2e14f37d5cdaea/1. pdf?pq-origsite $=$ gscholar\&cbl $=1006393$

15. Huarcaya-Victoria J. Consideraciones sobre la salud mental en la pandemia de covid-19. Rev Peru Med Exp Salud Pública [Internet]. 2020 [Citado 28/02/2021]; 37(2): 327-334. Disponible en: https://doi.org/10.17843/ rpmesp.2020.372.5419

16. Satizábal J. Polo L, Valbuena N. Teletrabajo Guía de Salud y Seguridad. Colombia: Exprecards CISAS; 2012

17. Swetha N, Shobha R, Ranganath T, Shibi $\mathrm{S}$, Nimra S. Estudio transversal de trastornos visuales y musculoesqueléticos entre los trabajadores profesionales de tecnología de la información en Bengaluru South, Karnataka, India. Revista Internacional de Medicina Comunitaria y Salud Pública [Internet]. 2016 [Citado 8/01/2021];3(10):2781-2785. Disponible en: $\quad$ http://dx.doi.org/10.18203/2394-6040. ijcmph20163361

18. Fernández $G$, Vizcaíno $F$, Llerena $L$, Baño F. Determinación de la fatiga ocular debido a teletrabajo en los docentes de la universidad UNIANDES de Ecuador. Dilemas contemp. educ. política valores [Internet]. 2021;8(3):1-20. https://doi.org/10.46377/dilemas.v8i3.2673

Conflicto de intereses. Los autores declaran que no existe conflicto de intereses para la publicación del presente artículo científico.

Financiamiento. Los autores declaran no recibieron financiamiento.

Agradecimiento. Los autores reflejan el esfuerzo y el aporte que las personas aportaron al desarrollo del presente artículo científico.

\section{ACERCA DE LOS AUTORES}

Silvia Elizabet Reyes Narváez. Maestría en salud de la mujer y el niño. Doctora en ciencias de enfermería en la Universidad Nacional de Trujillo. Docente, Universidad Nacional Santiago Antúnez de Mayolo. Miembro de la Sociedad Científica de Enfermería Pediátrica del Perú. Universidad Nacional Santiago Antúnez de Mayolo, Perú.

Llermé Nuñez Zarazú. Doctora en Enfermería. Magister en Educación Magíster en Salud Pública. Docente principal de Enfermería de la Universidad Nacional Santiago Antúnez de Mayolo. Investigadora RENACYT y CONCYTEC. Universidad Nacional Santiago Antúnez de Mayolo, Perú.

Bibiana María León Huerta. Doctora en Enfermería. Docente principal de la Facultad de Ciencias Médicas de la Universidad Nacional Santiago Antúnez de Mayolo. Decana de la Facultad de Ciencias médicas. Universidad Nacional Santiago Antúnez de Mayolo, Perú.

Ursula Lezameta Blas. Doctora en Enfermería. Docente Principal. Directora del Departamento Académico de Enfermería y miembro del Comité de Calidad de la Escuela Profesional de Enfermería de la Universidad Nacional Santiago Antúnez de Mayolo, Perú.

Olga Giovanna Valderrama Rios. Licenciada en Enfermería. Doctora en Enfermería. Miembro del Comité de Calidad de la UNAC Investigación en la línea de Salud Pública. Maestro en Salud Publica. Docente de la Universidad Nacional del Callao, Perú.

Ponte Valverde Segundo Ignacio. Magister. Jefe de la oficina de estadística de la empresa Agropecuaria Chiclin y Anexos S.A. Encargado del procesamiento de datos de estudios panel de la empresa TENPO marketig. Universidad San Ignacio de Loyola, Perú. 